\title{
Protocol optimization and evaluation of rice varieties response to in vitro regeneration
}

\author{
Puspasree Puhan*, Ebrahimali Abubacker Siddiq \\ Institute of Biotechnology, Acharya NG Ranga Agricultural University, Hyderabad, India \\ Email: *puspapuhan@gmail.com
}

Received 18 March 2013; revised 20 April 2013; accepted 12 May 2013

Copyright (C) 2013 Puspasree Puhan, Ebrahimali Abubacker Siddiq. This is an open access article distributed under the Creative Commons Attribution License, which permits unrestricted use, distribution, and reproduction in any medium, provided the original work is properly cited.

\begin{abstract}
Understanding the control of related molecular mechanisms is impossible without efficient and reproducible regeneration and stable genetic transformation. The key factors for enhancing successful regeneration are genotypes, tissue source of explants, combination and concentration of growth regulators, and culture conditions. In the present study different methods of regeneration were tested in a set of $\mathbf{1 2}$ rice accession representing indica, japonica, aromatic and wild groups. The highest frequency of shoot regeneration was achieved using mesocotyls derived from in vitro grown seeds cultured on Murashige and Skoogs, basal medium without growth regulators, when cultured on medium supplemented with Benzyl Adenine (BA) $0.5 \mathrm{mg} / \mathrm{l}$ under subdued light at $25^{\circ} \mathrm{C} \pm 2^{\circ} \mathrm{C}$. Under these conditions mesocotyls (Plurality of meristems) produced 3 to 4 tiller shoots in primary culture. One seed/One single mesocotyls segment produced over 5 to 9 shoots, arising primarily through direct organogenesis after 3 weeks of culture. Through callusing phase different rice cultivars produced different percentage of callusing however Basmati 370 gave high percentage in response to callussing, organogenesis and root formation. In between these two methods, the direct shoot regeneration gave $80 \%-100 \%$ result besides the wild species in respect with their genotype whereas the indirect organogenesis gave only $10 \%$ - $30 \%$ of plantlets. So the direct multiplication from mesocotyls is an efficient method for plantlet regeneration of rice cultivars through in vitro culture. Tissue culture derived plants when evaluated with rice markers no variability is found. For regeneration genotypes and its triggering factors like medium composition, culture conditions and type of explants are playing a combined role.
\end{abstract}

${ }^{*}$ Corresponding author.
Keywords: Primary Culture; Secondary Culture; Growth Regulator; Mesocotyls; Indirect Organogenesis

\section{INTRODUCTION}

Currently rice production supports more than $50 \%$ of the world population Rice consumers are increasing at the rate of $1.8 \%$ every year. It is estimated that rice production has to be increased $50 \%$ by 2025 [1]. Therefore, identification of useful genotypes for quick and efficient regeneration is a prerequisite for such genetic improvement of rice [2]. By using mature seeds for regeneration only certain varieties of rice such as Nipponbare (japonica) and Kasalath (indica) are responding well where as many of the popular varieties offer difficulty in both regeneration and transformation efficiency by a number of factors. This is proving a serious setback to development of targeted transgenic plants. Regeneration efficiency is affected by a number of factors including the genotype, the type and physiological status of the explants, composition of the culture medium, plant growth regulators and culture conditions [3,4]. Of these factors genotype and nutrient composition affect the variability in tissue culture [5-7].

Genetic engineering/Tissue culture is a potential tool to find solution to problems that defy conventional breeding approach due to either lack of needed native variability or/and reliable screening/selection strategies. Efficient regeneration protocol is the important prerequisite for successful application of genetic engineering for development of transgenic varieties of interest. Regeneration of plants by cell/tissue culture has been the challenging task across crop species especially monocots, the response being genotype and culture condition specific, rice is no exception.

From long experience of scientists it is evident that response to regeneration/transformation is genotype specific. The success of cell and tissue culture research depends upon reliable callus culture and plant regeneration 
procedures [8]. So identification of useful genotypes for quick and efficient regeneration is a prerequisite for such genetic improvement of rice [2]. Yet very little information is known about the genetic basis of it. It is required to indentify the reasons/ factors responsible for regeneration. Rice plants should receive all possible attention and efforts that contribute to conservation, including the use of plant tissue culture through in vitro micro propagation, high efficiency of regeneration to meet all the recent demands for rice cultivars. In the present study it is tried to standardize the in vitro regeneration protocol for rice both by direct and indirect method of organogenesis.

\section{MATERIALS AND METHODS}

Murashige and Skoog, 1962 (MS) [9] and Chu $1978\left(\mathrm{~N}_{6}\right)$ [10] salt composition, sucrose (30 g/l) and bacteriological grade agar-agar $(8 \mathrm{~g} / \mathrm{l})$ were used throughout the study with 2,4-D, Benzyl adenine (BA), Napthalic Acid (NAA), Caesin Hydrolysate, (CH), Indole-3 Acetic Acid (IAA), Indole-3 Butyric Acid(IBA), Adenine sulphate (ADS), Thiadizuron (TDZ) and also without any growth regulators were incorporated and the $\mathrm{pH}$ was adjusted 5.8. All the cultures were incubated in a culture room maintained at $25^{\circ} \mathrm{C} \pm 2{ }^{\circ} \mathrm{C}$ under $16 \mathrm{~h} / 8 \mathrm{~h}$ light/dark cycle, 45 $\mu \mathrm{mol} \cdot \mathrm{m}^{-2} \cdot \mathrm{s}^{-1}$ irradiance level provided by cool white fluorescent tubes (Philips, India) and with 55\% - 60\% RH. Each treatment consisted of 10/20/5 replicates and repeated thrice.

\subsection{Explant Preparation}

Seeds were dehusked with the help of forceps, needles and scalpels and washed in tap water and agitated in a liquid detergent solution for 15 - 20 minutes followed by agitation in $0.1 \% \mathrm{HgCl}_{2}$ for 7 - 8 minutes for surface sterilization. Finally the seeds were thoroughly rinsed in sterile double distilled water for at least four times under aseptic conditions and used as the source material for invitro germination.

\subsection{Media Preparation and Culture Condition}

Murashige Skoog,s salt composition, sucrose (30 g/l) and bacteriological grade agar-agar (8 g/l) were used throughout the study without any growth regulators were incurporated and the $\mathrm{pH}$ was adjusted 5.8. All the cultures were incubated in a culture room maintained at $25^{\circ} \mathrm{C} \pm$ $2^{\circ} \mathrm{C}$ under $16 \mathrm{~h} / 8 \mathrm{~h}$ light $/$ dark cycle, $45 \mu \mathrm{mol} \cdot \mathrm{m}^{-2} \cdot \mathrm{s}^{-1} \mathrm{ir}-$ radiance level provided by cool white fluorescent tubes (Philips, India) and with 55\% - 60\% RH. Each treatment consisted of 10 replicates and repeated thrice.

\subsection{Subculture of Micro-Shoots for Rooting}

Proliferated micro-shoots were separated and those meas- uring 2 - $3 \mathrm{~cm}$ and above were individually planted onto the basal MS medium without the supplementation of auxins for rooting.

\subsection{Acclimatization, Hardening and Potting of Propagules and Field Establishment of the Regenerated Plants}

Plantlets with healthy root systems were washed (especially the root portions) under running tap water to clear off the entire residual agar medium so as to check the chances of contamination in soil. The root portions were then dipped for about 4 - 15 minutes in an antifungal solution $(0.2 \%$ or $0.1 \%(\mathrm{w} / \mathrm{v}) \mathrm{w} / \mathrm{v}$ "Bavistin", Bayer India). The plantlets were then transferred to root trainers or transplanted in small ( $5 \mathrm{~cm}$ diameter) plastic tea cups containing pre-soaked sterilized vermiculite medium and kept inside the mist chamber. The timer was set to 1 minute on time and 30 minutes off time and the relative humidity was set to $80 \%$ - 90\%. After recording the data, the propagules were carefully planted in polybags (of the size $20 \mathrm{~cm} \times 10 \mathrm{~cm}$ ) filled with autoclaved sand, soil/red earth and farmyard manure (v:v:v) in the proportions of 1:1:1/1:2:1/2:2:1. The poly bags were fertilized with $5 \mathrm{ml}$ of $1 / 4$ strength MS major and minor salts on alternate days for the first week and then watering was done as and when required. Then they were kept under the shade house conditions for further period of two weeks or hardened under a polyethylene tent in the greenhouse for at least one-two weeks. The green house was maintained with a humidity of $85 \%$ relative humidity at $28^{\circ} \mathrm{C}-30^{\circ} \mathrm{C}$ under daylight conditions. Each treatment was replicated 10 times. After hardening for about 7 - 10 days in the polythene bags, the plants were transferred into open light conditions and maintained in the experimental gardens or transferred to bigger earthen pots $(25 \mathrm{~cm}$ diameter) containing a non-sterile sand: soil: compost mixture (1:3:1). When the new sprouts were formed, they were transplanted in the field.

\subsection{Observations, Collection, Scoring and Statistical Treatment of Data}

All the data observed, collected and documented on multiplication and rooting were statistically analyzed by mean, standard error and some of the results were compared by DMRT [11]. Results are presented in mean \pm SE of three independent experiments each with 20/15/10/5 replicates in order to find out the accuracy of results.

The different stages of in vitro development of shoots, regenerants, rooting and in vivo establishment of the plantlets were photographed with the help of one "EPSON" (3.3 mega pixel) digital camera as the record of evidence. 


\subsection{Evaluation with Rice Primers}

The SSR primers/rice markers covering the entire 12 chromosome were taken to see the variation existed between normal and tissue culture derived plants. The lists of primers taken are listed below for one to twelve chromosomes.

\subsection{PCR Primers for Amplification of SSR Markers}

Each PCR reaction was performed in $25 \mathrm{ml}$ (total volume) of reaction mixture consisting of approximately $50 \mathrm{ng}$ of genomic DNA from normal as well as tissue culture grown plants, $1 \times$ PCR buffer, $50 \mu \mathrm{M}$ dNTPs, $1.5 \mathrm{mM}$ $\mathrm{MgCl}_{2}, 5$ pmoles of each primer and 1 unit of Taq DNA polymerase. PCR was carried out in a thermal cycler (ABI Veriti) under the following conditions: $94^{\circ} \mathrm{C}$ for 4 min as preheating, then 35 cycles of $94^{\circ} \mathrm{C}$ denaturing for $1 \mathrm{~min}, 55^{\circ} \mathrm{C}$ annealing for $1 \mathrm{~min}$ and $72^{\circ} \mathrm{C}$ extension for $1 \mathrm{~min}$ and $10 \mathrm{~min}$ at $72^{\circ} \mathrm{C}$ final extension. Amplified
DNA fragments were electrophoresed on $1.2 \%$ agarose gel and detected by ethidium bromide staining and photographed under ultraviolet light of Bio-Rad Molecular Imager Gel Doc XR System for SSR markers.

\subsection{Variation}

The variation for similarity and clustering was analyzed by the software NT-SYS-PC (2.0 versions).

\section{RESULT}

\subsection{Standardisation of Germination and Multiplication Protocol: Direct Multiplication}

Dehusked rice seeds of twelve varieties viz., Jaya, IR-64, AC 37538 (indica), Basmati370, PusaBasmati-EIC-005 (aromatic), AC 41039, 40774, 41036, 40778 (japonica) and IC 20339, IC 277296, IC 277286 (wild) were surface sterilized and germinated on MS basal medium (Table 1, Figures 1(f)

Table 1. Rice markers (RM) on chromosomes (Chr.) 1 - 12.

\begin{tabular}{cccccccccccc}
\hline Chr. 1 & RM562 & Chr. 2 & RM13131 & Chr. 3 & RM14778 & Chr. 4 & RM16577 & Chr. 5 & RM18384 & Chr. 6 & RM20037 \\
\hline & RM12353 & & RM13584 & & RM14735 & & RM17669 & RM5907 & RM20710 \\
Chr. 7 & RM21693 & Chr. 8 & RM22554 & Chr. 9 & M23741 & Chr. 10 & RM5708 & Chr. 11 & RM26190 & Chr. 12 & RM27840 \\
& & & RM22658 & & RM240044 & & RM25262 & RM25969 & RM6965 \\
\hline
\end{tabular}

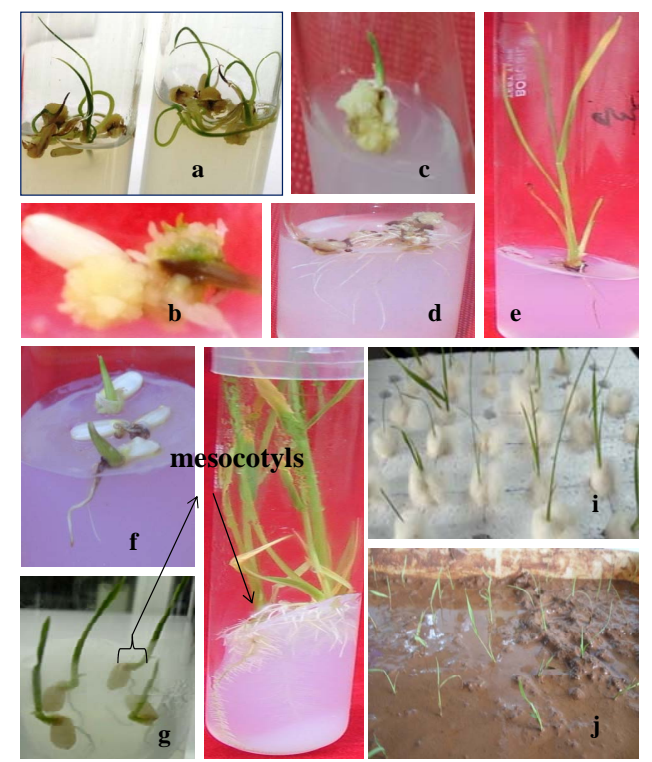

Figure 1. (a) Germinated seeds on MS with 2,4-D $0.5 \mathrm{mg} / \mathrm{l}$ (after one week of culture); (b) Callusing from mesoctyls; (c) Callus giving shoots on regeneration medium; (d) Callus giving only roots; (e) Rooting of a callus derived shoot; (f) and (g) Germinated seeds on MS medium after 4 days and 1 week of culture; (h) Mesocotyl meristemoids giving multiplied shoot and root simultaneously on MS with $0.5 \mathrm{mg} / \mathrm{l} \mathrm{BA}$; (i) Hardening of callus derived shoots; (j) Hardening of shoots derived from mesocotyl meristemoids. 
and (g)). Two to three numbers of primary tillers formed after two weeks of culture. Then the primary tillers after two weeks were cut and placed in the rooting medium leaving the mesocotyls with multiple meristem. For its secondary passage Mesocotyls grown on MS with 0.5 mg/l BA (multiplication medium-MM). Again 2 - 4 secondary tillers with well developed roots (Figure 1(h)) produced.

Of different medium with various hormone composition tried for in vitro seed germination and plant multiplication of mesocotyl explants derived from germinated rice seeds, MS medium without any hormone supplement was found to be the best. Mesocotyls from germinated seeds of when cultured on N6 and MS medium with different hormones like BA, Kinetin (Kn) alone or in combination (Data with N6 and AR medium has not given), the MS medium containing $0.5 \mathrm{mg} / \mathrm{l} \mathrm{BA}$ is proved to be the best for multiplication of shoots from mesocotyl explants and produced three to five shoots from all varieties. Among the varieties tried, Basmati 370 and EIC-005 (Pusa Basmati) responded comparatively with higher shoot multiplication than other three varieties (Table 2). Plain MS and MS with BA supplement proved effective and rewarding in terms of direct shoot multiplication.

\subsection{Indirect Organogenesis/Somatic Embryogenesis}

Again mature seeds of rice varieties were taken for standardization of the protocols for in vitro plant regeneration through somatic embryogenesis from callus induced. Basal media, namely AR, N6 and MS with different formulation of 2,4-D, BA, CH, ADS, TDZ and NAA were tested for callus induction; of these MS was found best for callus induction with $0.5 \mathrm{mg} / \mathrm{l}$ 2,4-D (Callus induction medium or CIM medium) and for regeneration with and without $0.5 \mathrm{mg} / \mathrm{l} \mathrm{BA}$ in $1 / 2 \mathrm{MS}$ basal medium (Regeneration medium or RM medium). The aromatic variety was found most responsive and degree of callusing is normalhigh in some culture tubes on CIM medium. The best callus induction period was 4 weeks (Figures 1(a) and (b)). Results also showed that callus transferred into organogenesis induction medium gave the highest regeneration frequency $(40 \%-50 \%)$ in both the cultivars, when transferred to RM, the regeneration medium. Some cultures produced only roots (Figure 1(d)), only shoots with basal callus (Figure 1(c)), greenish nodular heads like structures. The total duration of plant regeneration protocol from inoculation till rooted plantlets was ready for transfer to green house was 15 weeks (Table 3). From the results it is found that aromatic variety is highly responsive and IR64 among the indica variety is moderate and other indica and japonica variety are less moderate to moderate while the wild varieties are non responsive in both direct and indirect organogenesis method. But the cultivated varieties irrespective of their genotype are showing very good response to direct multiplication from mesocotyls.

\subsection{Rooting}

Then the individual shoots derived from primary tillers, secondary tillers and from callus which were cut at the base and grown on the same MS medium, produced two to three well developed roots (Figure 1(e)).

\subsection{Hardening}

After 4weeks of culture the rooted plantlets were thoroughly washed with tap water and dipped in an antifungal solution $0.1 \%(\mathrm{w} / \mathrm{v})$ Bavistin solution for 5 minutes, then washed and planted in plastic pots containing autoclaved artificial soil and were supplied sterile distilled water for 4weeks. Established plants were repotted in polythene bags, then to bigger earthen pots (Figures 1(i) and (j)) and kept under shade ( $40 \%$ shade) in the garden for another 3 weeks and watering was done as and when required. When the plants were established, they were transferred to open light conditions.

\subsection{Treatment with SSR Markers}

Varieties when treated with rice markers no remarkable difference is found (Figures 2(a) and (b)).

\subsection{Comparison of Two Methods}

When direct and indirect method for multiplication compared it is found that the first one is superior to organogenesis through callusing in terms of response, time requirement, number of shoots produced and success rate in field trial. The plants derived from mesocotyls showed $100 \%$ field establishment with greenish leaves the sign of healthy growth but the callus derived plants after two weeks of transfer to pots started drying and only 30\% $40 \%$ plant survived.

\section{DISCUSSION}

In the present study it is tried to identify optimal culture conditions required for regeneration across varieties. Hence Plant tissue culture system is vital for successful crop improvement. The ability of plants to regenerate is essential for establishing a successful plant culture system. However, not all crop species or varieties can regenerate easily. Generally, it is difficult to culture and regenerate agronomically important crops such as rice, wheat, and maize.

For 12 varieties from indica, japonica, javonica, aromatic and wild varieties we tried to develop highly reproducible methods for in vitro multiplication. Besides the wild, all varieties responded well in direct multiplication from mesoctyls meristemoids. So not only genotype but also culture conditions, explants types and methods 


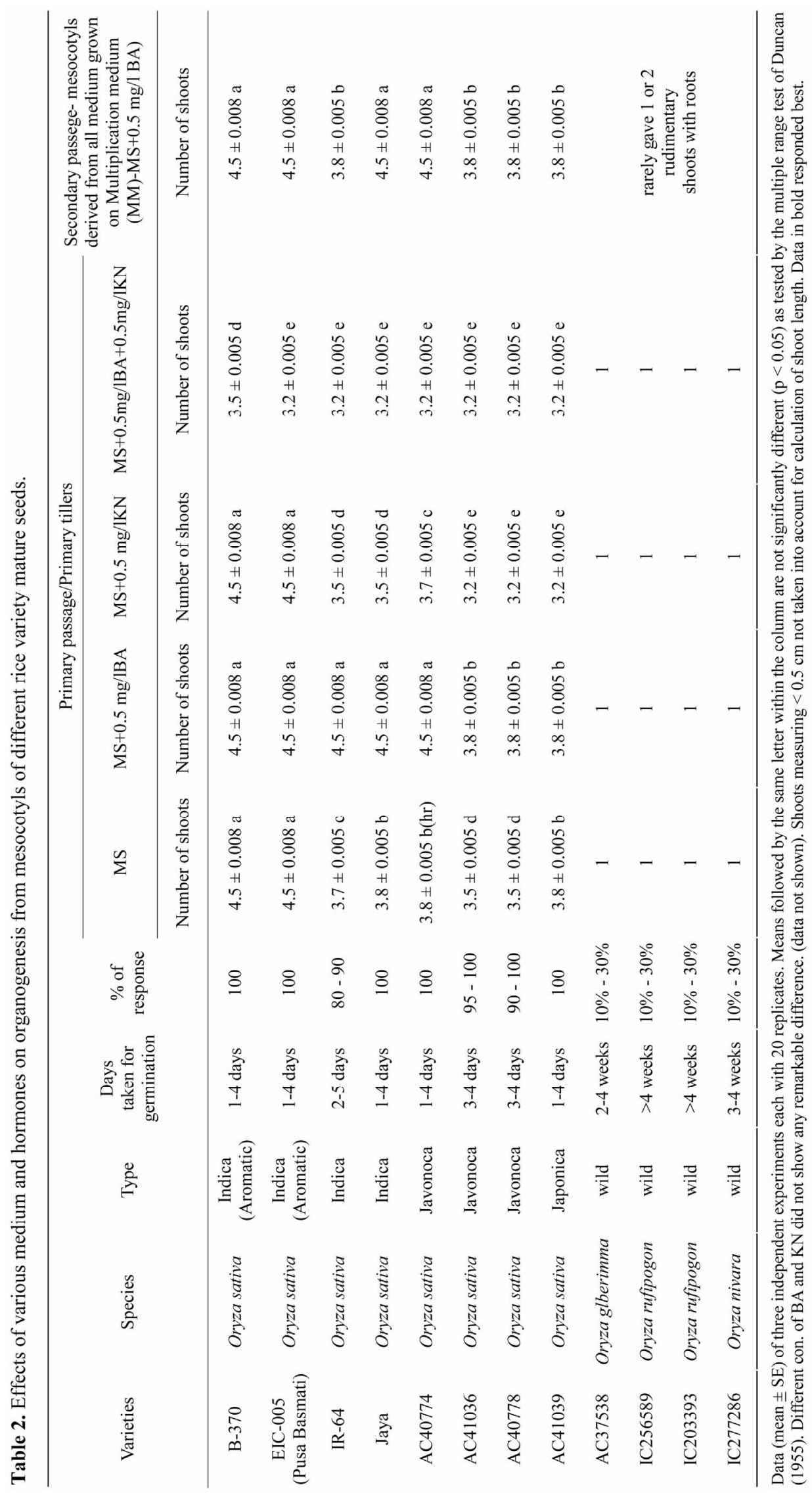


Table 3. Effects of various medium and hormones on callussing and organogenesis from mesocotyls of different rice variety mature seeds.

\begin{tabular}{|c|c|c|c|c|}
\hline Callussing & & $\%$ of response & Degree of callusing response & Response in regeneration medium (MS and $\mathrm{MS}+0.25 \mathrm{mg} / \mathrm{l} \mathrm{BAP}$ ) \\
\hline Varieties & \multirow{13}{*}{ 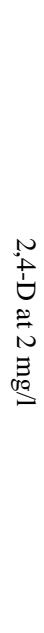 } & & & \\
\hline B-370 & & $40 \%-80 \%$ & (normal) & gnh, ap and rs, rarely 1 - 2 gs, only rt (RF-40-50\%) \\
\hline EIC-005(Pusa Basmati) & & $30 \%-70 \%$ & (normal) & gnh, ap and rs, rarely 1 - 2 gs, only rt (RF40-50\%) \\
\hline IR-64 & & $30 \%-60 \%$ & (moderate) & gnh, ap and rs, rarely 1 - 2 gs, only rt (RF20-40\%) \\
\hline Jaya & & $20 \%-60 \%$ & (moderate) & gnh, ap and rs, rarely 1 - 2 gs, only rt (RF 20-30\%) \\
\hline AC40774 & & $30 \%-70 \%$ & $(<$ moderate $)$ & gnh, ap and rs, rarely 1 - 2 gs, only RT (RF20-30\%) \\
\hline AC41036 & & $30 \%-70 \%$ & $(<$ moderate $)$ & gnh, ap and rs, rarely 1 - 2 gs, only rt (RF20-30\%) \\
\hline AC40778 & & $30 \%-70 \%$ & (<moderate) & gnh, ap and rs, rarely 1 - 2 gs, only RT (RF40-50\%) \\
\hline AC41039 & & $30 \%-70 \%$ & (normal) & gnh, ap and rs, rarely 1 - 2 gs, only rt (RF40-50\%) \\
\hline AC37538 & & $1 \%-2 \%$ & (slow or nill) & \multirow{4}{*}{$\begin{array}{l}\text { in all of the cases browinsh callus (RF10\%) } \\
\text { with very fine and reduced roots }\end{array}$} \\
\hline IC256589 & & $1 \%-2 \%$ & (slow or nill) & \\
\hline IC203393 & & $1 \%-2 \%$ & (slow or nill) & \\
\hline IC277286 & & $1 \%-2 \%$ & (slow or nill) & \\
\hline
\end{tabular}

RF-regeneration frequency, gnh—greenish nodular heads, ap—albino plants, rs—rudimentary shoots, gs—greenish shoots, rt—roots. The degree of callusing is visualized and if it requires sub culturing within 2 - 3 weeks then it is normal, if it is 4 - 5 weeks it is moderate and if it is 5 - 6 weeks then less moderate, if it is more than 1 month then it is slow or nil growth.

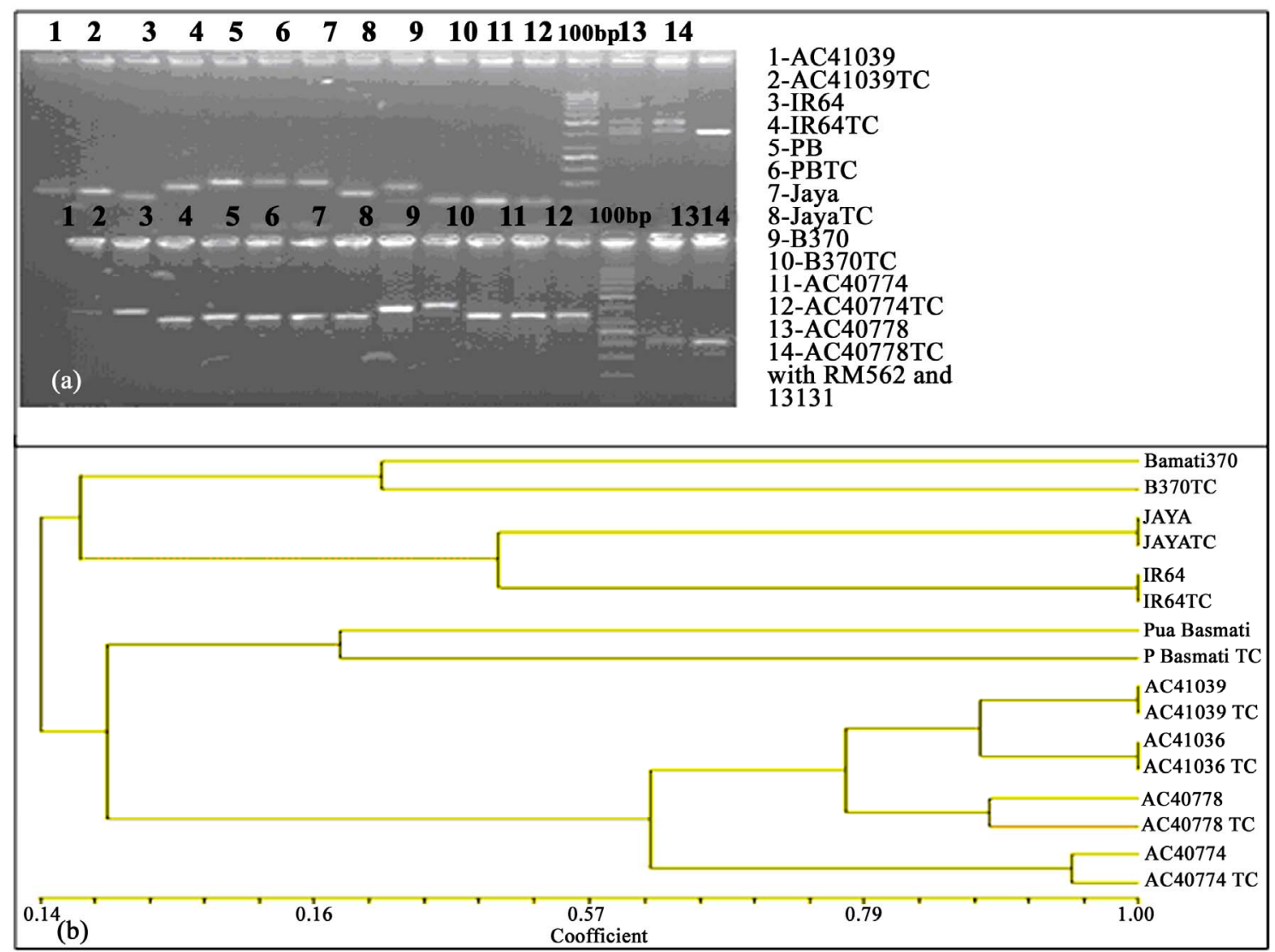

Figure 2. (a) 1-AC41039, 2-AC41039TC, 3-IR64, 4-IR64TC, 5-PusaBasmati, 6-PusaBasmatiTC, 7-Jaya, 8-JayaTC, 9-Basmati370, 10-Basmati370TC, 11-AC40774, 12-AC40774TC, 13-AC40778, 14-AC40778TC with RM562 and 13131 (TC-Tissue culture derived); (b) The variation for similarity and clustering analyzed by the software NT-SYS-PC (2.0 versions). 
are playing greater role in regeneration process. In rice, an efficient culture system using mature seeds has been established for some model varieties such as Nipponbare (Japonica) and Kasalath (Indica) [12].

All the genotypes representing three major subspecies of rice tried for protocol development did not show any significant difference in tissue culture response when analyzed by rice markers for similarity and clustering suggesting that the regeneration protocol developed through direct organogenesis from mesocotyls meristemoids derived from mature seeds can be efficiently used across the two major subspecies/ecotypes of the Asian rice cultivar Oryza sativa. In this method mature seeds are found as best explants for regeneration protocol usable across a variety of genotypes through in vitro clonal propagation/shoot bud multiplication from mesocotyls avoiding the widely dependent on callus-embryogenic route which is controlled by many physiological and genetic factors like NiR (nitrate reductase gene) activity in transgenic research [12]. The protocol outlined offers a potential strategy for $100 \%$ regeneration efficiency in rice which can be a best method for large scale propagation of rice-a high food value cereal [13].

It is observed that explants played an important role in regeneration. So the tissue culture maneuverability i.e. explants, culture condition and medium manipulation playing a greater role in regeneration $[13,14]$. Direct multiplication from mesocotyl explants from mature seeds can be used universally for transgenic production in rice.

\section{REFERENCES}

[1] Khush, G.S. and Virk, P.S. (2000) Rice breeding: Achievement and future strategies. Crop Improvement, 27, 115144.

[2] Hoque, E.H. and Mansfield, J.W. (2004) Effect of genotype and explants age on callus induction and subsequent plant regeneration from root-derived callus of indica rice genotypes. Plant Cell, Tissue and Organ Culture, 78, 217-223. doi:10.1023/B:TICU.0000025640.75168.2d

[3] Rueb, S., Leneman, M. Schilperoort, R.A. and Hensgens, L.A.M. (1994) Efficient plant regeneration through embryogenesis from callus induced on mature rice embryos (Oryza sativaL.). Plant Cell, Tissue and Organ Culture, 36, 259-264. doi:10.1007/BF00037729
[4] Ge, X., Chu, Z. Lin Y. and Wang, S. (2006) A tissue culture system for different germplasms of indica rice. Plant Cell Reports, 25, 392-402. doi:10.1007/s00299-005-0100-7

[5] Khanna, H.K. and Raina, S.K. (1998) Genotype x culture media interaction effects on regeneration response of three indica rice cultivars. Plant Cell, Tissue and Organ Culture, 52, 145-153. doi:10.1023/A:1006032303195

[6] Al-Forkan, M., Rahim, M.A. Chowdhury, T. Akter, P. and Khaleda, L. (2005) Development of highly in vitro callogenesis and regeneration system for some salt tolerant rice (Oryza sativa L.) cultivars of Bangladesh. Biotechnology, 4, 230-234. doi:10.3923/biotech.2005.230.234

[7] Kunanuvatchaidach, R., Godwin, I.D. and Adkins, S.W. (1995) High efficiency plant regeneration from callus induced on mature indica rice caryopses. Australian Journal of Botany, 43, 337-348. doi:10.1071/BT9950337

[8] Sugimoto, K., Miyake, H. and Takiyoka, Y. (2000) Genetic diversity of regeneration ability of Anther culture of rice (Oryza sativa L). Plant Production Science, 3, 387391. doi:10.1626/pps.3.387

[9] Murashige, T. and Skoog, F. (1962) A revised medium for rapid growth and bioassay with tobacco tissue culture. Physiologia Plantarum, 15, 473-497. doi:10.1111/j.1399-3054.1962.tb08052.x

[10] Chu, C.C. (1978) The N6 medium and its applications to anther culture of cereal crops. In: Proceedings of Symposium on Plant Tissue Culture, 25-30 May 1978, Science Press, Peking, 45-50.

[11] Duncan, D.B. (1955) Multiple range and multiple $F$ tests. Biometrics, 11, 1-42. doi:10.2307/3001478

[12] Nishimura, A., Ashikari, M. Lin, S. Takashi, T. Angeles, E.R. Yamamoto, T. and Matsuoka, M. (2005) Isolation of a rice regeneration quantitative trait loci gene and its application to transformation systems. Proceedings of the National Academy of Sciences of USA, 102, 11940-11944. doi:10.1073/pnas.0504220102

[13] Puhan, P., Vipparla, A. Vemireddy, L.R. Anuradha, G. and Siddiq, E.A. (2012) An efficient and universal Agrobacterium-mediated transformation protocol in rice. Journal of Plant Biochemistry and Biotechnology, 21, 252260.

[14] Visarada, K.B.R.S. and Sharma, N.P. (2002) Qualitative assessment of tissue culture parameters useful in transformation of indica rice. Current Science, 82, 343-347. 